\section{Protección financiera en salud: evolución y comparación del gasto de bolsillo de los hogares chilenos}

\author{
EDUARDO GALLEGOS ${ }^{1, \mathrm{a}}$, ALBERTO MUÑOZ ${ }^{2, \mathrm{~b}}$
}

\section{Financial protection for health care expenses provided by public and private systems in Chile}

\begin{abstract}
Background: People need a financial protection system to face the high costs of health care. Aim: To compare the financial protection between households affiliated to the Chilean public health financing system (FONASA) or to a private health financing system (ISAPRE). To describe the evolution of protection at the national level between 2007 and 2012. Material and Methods: As proposed by the World Bank, impact indicators to measure the equity and efficiency of the insurance systems were generated. Namely, average out-of-pocket expenses by insurance and average out-of-pocket spending as a percentage of expenditure. Also, the evolution of out-of-pocket spending by quintiles and Gini Coefficient were measured as measure of equity. To determine these, Family Budget Surveys for 2007 and 2012 were used. Results: Household out-of-pocket spending increased by $14.12 \%$. When expressed as a percentage of total expenditure, it grew from $5.6 \%$ to $6.2 \%$. Household Gini coefficient and per capita out-of-pocket spending decreased and the ratio between the highest and lowest quintile out-of-pocket increased at both analysis levels. Pocket expense in absolute values or expressed as a percentage of total expenses was higher among persons insured in private systems than those affiliated to the public financing service. Conclusions: Outof-pocket spending increased for all income groups and people insured in the public system had a lower absolute and relative spending than those insured in private systems.
\end{abstract}

(Rev Med Chile 2018; 146: 737-744)

Key words: Family Characteristics; Health; Health Expenditures; Insurance.
'Escuela de Administración y Economía, Facultad de Ciencias Sociales, Jurídicas y Económicas, Universidad Católica Silva Henríquez. Santiago, Chile. ${ }^{2}$ Instituto de Administración en Salud, Facultad de Economía y Negocios, Universidad de Chile. Santiago, Chile.

Ingeniero Comercial, Magister en Políticas Públicas.

bIngeniero Comercial, Master of Arts in Economics.

Los autores declaran no tener conflictos de interés. Trabajo no recibió

financiamiento.

Recibido el 27 de junio de 2017, aceptado el 11 de mayo de 2018.

Correspondencia a: Eduardo Gallegos, San Isidro 337, Depto. 1317. Santiago, Chile. eandresgc@hotmail.com
$\mathrm{E}$ l sistema de salud de Chile, para aquella población que no forma parte de las fuerzas armadas ${ }^{1,2}$, es principalmente dual y segmentado, conformado por un régimen solidario público, a cargo del Fondo Nacional de Salud (FONASA) y un régimen individual privado, a cargo de las Instituciones de Salud Previsional (ISAPRE) $)^{3,4}$. Para el año 2014, el FONASA aseguraba al $75,2 \%$ de la población, personas de menores ingresos, más edad y una mayor proporción esperada de enfermos crónicos ${ }^{5}$, mientras que las
ISAPRE atienden a $18,5 \%$ restante $^{6}$ compuesta por personas de altos ingresos, preferentemente hombres entre 25 y 45 años, y bajos riesgos esperados $^{5}$. Conformando un sistema segmentado por riesgo e ingreso ${ }^{3}$.

Una de las funciones principales de un seguro de salud es proteger a las personas de las consecuencias monetarias del gasto asociado a un evento de salud ${ }^{7-9}$. Es decir, ya sea público o privado, deberían asegurar contra los costos del tratamiento asociado a eventos adversos en la 
salud de los individuos ${ }^{10}$. Esta función es conocida como protección financiera de los seguros de salud $^{11}$, la que a su vez puede ser medida a través de los indicadores de impacto -permiten obtener una imagen de los resultados intermedios o finales de un seguro de salud-, propuestos por el Banco Mundial (BM) $)^{9}$, que considera comparar en términos de equidad, eficiencia y eficacia los distinto seguros de salud ${ }^{12}$.

Desde el año 2010, la Organización Mundial de la Salud $(\mathrm{OMS})^{8}$ promueve la cobertura universal, con el doble objetivo de garantizar el acceso a los servicios de salud y proteger del riesgo financiero a los individuos que accedan a dichos servicios, aumentando de esta forma la probabilidad de obtener mejores resultados sanitarios. El riesgo financiero estaría determinado por la proporción de los costos que los individuos pagan directamente de su bolsillo, los que bajo una cobertura universal no deberían exceder un umbral; menores a $20 \%$ de los gastos de bolsillo (GB) sobre los gastos totales en salud ${ }^{13}$ y para los más pobres debería ser cero ${ }^{8}$.

Para la $\mathrm{OMS}^{8}$ y el Instituto Nacional de Estadísticas (INE) ${ }^{14}$ se entiende el GB como los desembolsos directos que realizan los hogares por todas las prestaciones de salud, incluyendo los gastos en copago, los adicionales al 7\% obligatorio y el efectuado en seguros complementarios. Sin embargo, en esta investigación se consideró como un acercamiento del GB el gasto destinado a copagar las atenciones. Este se entiende como el valor total de la prestación de salud menos la cobertura del seguro.

La presente investigación tiene por objetivo primario comparar la protección financiera entre hogares afiliados a FONASA y a ISAPRE para el año 2012, solo para este período se disponía de dicha apertura, y, como objetivo secundario, describir la evolución de la protección financiera a nivel nacional (NN), entre los años 2007 y 2012, sin diferenciar por seguros, en términos de equidad y eficiencia, mediante el análisis del GB.

\section{Material y Método}

La comparación de la protección financiera entre los seguros se efectuó, para el año 2012, a través del cálculo del GB promedio por hogar (indicador 1) y per cápita (PC) (indicador 2), además de los GB como porcentaje de los gastos por hogar (indicadores 3 y 4 ).
Por su parte, la evolución se estableció a través del GB promedio por hogar (indicador 5) y PC (indicador 6) y el GB como porcentaje del gasto total por hogar (indicadores 7 y 8), así como los índices de GINI (IG) del GB promedio por hogar (indicador 9) y PC (indicador 10), ambos por quintiles de ingreso, y la relación entre el quintil V y I en GB promedio por hogar y PC. Estos indicadores se determinaron de manera general, sin distinción de seguro de salud, comparando el año 2007 y 2012.

Los indicadores utilizados fueron:

(1) Gasto de Bolsillo Promedio por Hogar por Seguro

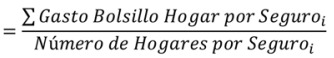

(2) Gasto de Bolsillo Promedio Per Cápita por Seguro $_{i}$

$$
=\frac{\sum\left(\frac{\text { Gasto Bolsillo Hogar por Seguro }{ }_{i}}{\text { Número de Personas por Hogar por Seguro }}\right)}{\text { Número de Ho gares por Seguro } o_{i}}
$$

(3) Gasto de Bolsillo Promedio Ho gar como Porcentaje de los Gastos Totales Hogar por Seguro ${ }_{i}$

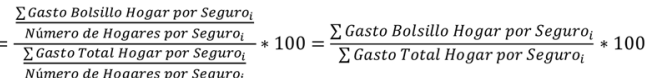

(4) Gasto de Bolsillo Promedio Per Cápita como Porcentaje de los Gastos Totales Hogar por Seguro ${ }_{i}$

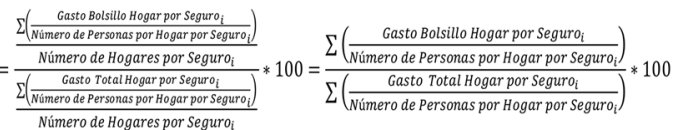

Donde $i$ toma los valores de FONASA e ISAPRE calculados a NN, Gran Santiago (GS), Resto Capitales Regionales (RCR), Jefe de Hogar Hombre (JHH) y Jefa de Hogar Mujer (JHM).

(5) Gasto de Bolsillo Promedio por Hogar $r_{t}=\frac{\sum \text { Gasto Bolsillo Hogar }}{\text { Número de Hogares }}$

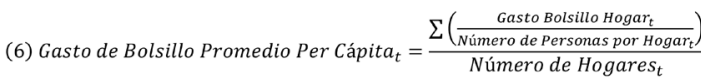

(7) Gasto de Bolsillo Promedio Hogar como Porcentaje de los Gastos Totales Hogar

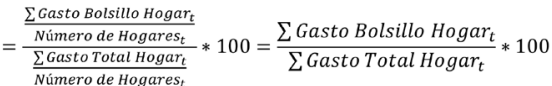

(8) Gasto de Bolsillo Promedio Per Cápita como Porcentaje de los Gastos Totales Per Cápita

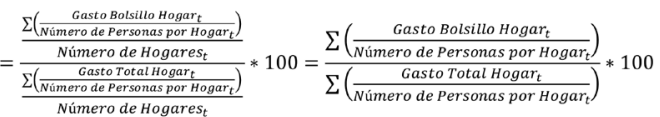

Donde $t$ toma los valores del período de análisis, calculados a NN, GS y RCR. 
(9)

Gini Gasto de Bolsillo Promedio por Hogar

$$
=\left|1-\sum_{i=1}^{n-1}\left(X_{i+1, t}-X_{i, t}\right)\left(G B_{i+1, t}+G B_{i, t}\right)\right|
$$

Donde $X$ es la proporción acumulada de hogares y $G B$ es la proporción acumulada del $G B$ promedio por hogar, en el período $t$.

(10) Gini Gasto de Bolsillo Promedio Per Cápita

$$
=\left|1-\sum_{i=1}^{n-1}\left(X_{i+1, t}-X_{i, t}\right)\left(G B P C_{i+1, t}+G B P C_{i, t}\right)\right|
$$

Donde $X$ es la proporción acumulada de hogares y GBPC proporción acumulada del GB promedio per cápita, en el período $t$. El indicador fluctuaría entre 0 (perfecta igualdad) y 1 (máxima desigualdad).

Para determinar los indicadores se utilizó la Encuesta de Presupuestos Familiares (EPF) 2007 y $2012^{14}$, cuyo objetivo es identificar la estructura y características del gasto en el consumo final de los hogares. La EPF 2007 y 2012 comprenden una muestra de 10.096 y 10.528 hogares, respectivamente. La última realiza una apertura por seguros de salud (64,94\% de FONASA y el resto a ISAPRE) y presenta un error relativo de estimación de los gastos para el GS y el RCR, de 10,42\% y de 6,17\%, respectivamente ${ }^{15}$.

La EPF no fue diseñada para la obtención de información de prestaciones de salud, por lo que puede presentar problemas de representación y de estimación en los gastos de salud, no obstante, es la única fuente de datos disponible para realizar este tipo de investigaciones. Para este análisis se consideraron los siguientes alcances metodológicos:

Se consideró a todos los hogares, independientemente de si registraran copagos por prestaciones de salud.

La variable GB promedio por hogar se construyó con la sumatoria de los copagos por prestaciones de salud efectuado por todos los individuos de un hogar.

La evolución de la protección financiera considera el GB de 2007 y 2012 por hogar y PC, a NN, GS y RCR.

Para analizar la equidad se utilizó el cálculo por quintiles (ordenados en base a los ingresos totales PC, metodología utilizada por el INE) y el IG.
Las potenciales brechas estadísticas de género entre seguros, se analizaron a través del GB por hogar de la persona jefe (a) de hogar.

Finalmente, se obtuvo la significancia estadística respecto a las diferencias de los GB por seguro en términos absolutos, utilizando un test de medias (Test Ajustado Wald, de distribución $\left.F^{16}\right)$. El nivel de significancia empleado para rechazar las hipótesis alternativas consideró un $\mathrm{p}$ value $<0,05$.

\section{Resultados}

\section{Protección financiera entre seguros}

En cuanto a la medición de la eficiencia entre los seguros (Tabla 1), a nivel de hogares y PC los asegurados por las ISAPRE realizaron, estadísticamente, un GB promedio mayor a los hogares afiliados a FONASA ( $\$ 124.574$ y $\$ 33.169$ hogar y $\$ 44.951$ y $\$ 11.810$ PC, respectivamente por seguro).

Si el análisis es por procedencia geográfica del hogar, el GB realizado por los hogares afiliados a las ISAPRE, en comparación con los afiliados a FONASA, es estadísticamente mayor tanto en el GS (4,4 veces hogar y 4,7 PC) como en el RCR (3,0 veces por hogar y 2,9 PC). Por otra parte, comparando la procedencia geográfica por seguro, los resultados muestran que los afiliados al FONASA poseen un GB por hogar y PC estadísticamente menor en el GS que en el RCR, a diferencia con los asegurados en las ISAPRE que presentan un mayor gasto PC en el GS (Tabla 1).

Al comparar la situación entre JHH y JHM, el GB por hogar en ISAPRE es mayor 3,8 y 3,9 veces, mientras que el gasto bolsillo $\mathrm{PC}$ es mayor en ISAPRE 4,0 y 3,9 veces, respectivamente, en comparación con los afiliados a FONASA. No hubo diferencias significativas entre JHH y JHM al analizar el GB hogar por seguro, pero en el análisis PC encontramos un GB mayor para las JHM tanto en afiliados a FONASA como en ISAPRE (Tabla 1).

En términos descriptivos (Tabla 2), el GB como porcentaje de los gastos totales tanto por hogar y PC, es de 5,7\% y de 6,8\% para los afiliados a FONASA e ISAPRE, respectivamente. El menor porcentaje de GB como porcentaje del gasto total de los asegurados por el FONASA con respecto a los afiliados a las ISAPRES, se repite al comparar por zonas geográficas y por género del jefe de 
Tabla 1. Gasto de bolsillo en salud (hogar y per cápita) por seguros año 2012 (\$ de 2015)

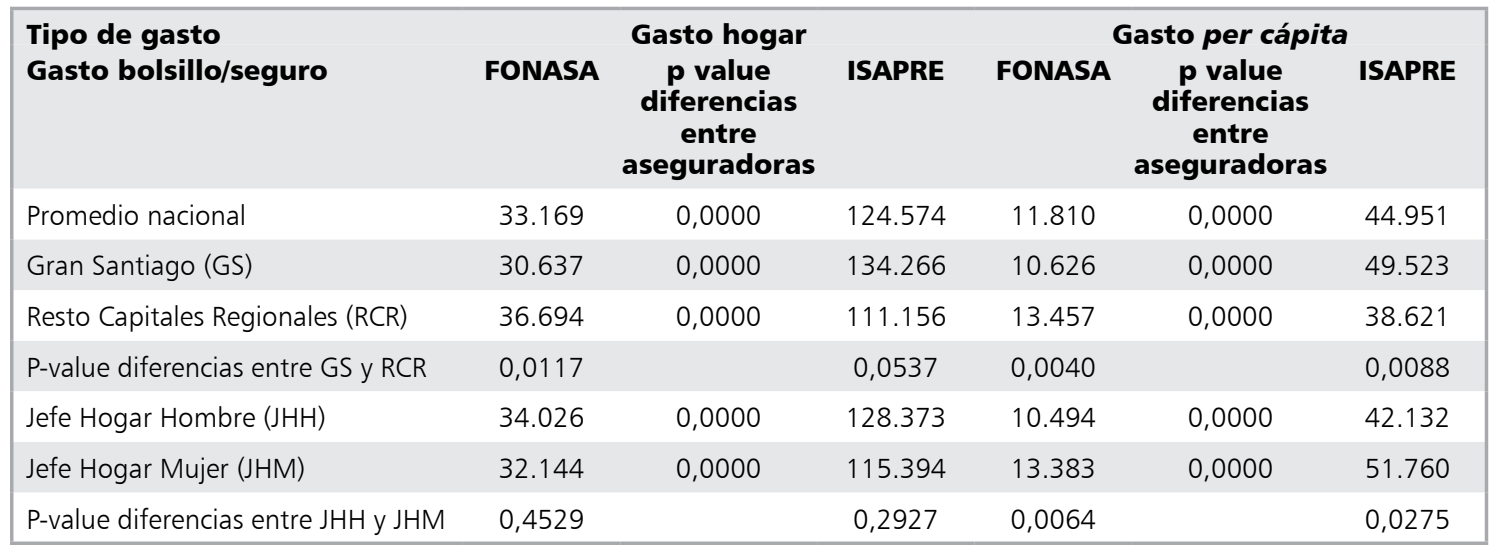

Fuente: Elaboración propia, con datos de la Encuesta Presupuestos Familiares, del año 2012.

Tabla 2. Gasto de bolsillo en salud (hogar y per cápita) como porcentaje de los gastos totales por seguros año 2012

\begin{tabular}{|lcccc|}
\hline Tipo de gasto & \multicolumn{2}{c}{ Gasto hogar } & \multicolumn{2}{c|}{ Gasto per cápita } \\
Gasto bolsillo/seguro & FONASA & ISAPRE & FONASA & ISAPRE \\
\hline Promedio nacional como \% de los gastos totales & $5,70 \%$ & $6,80 \%$ & $5,70 \%$ & $6,80 \%$ \\
\hline GS como \% de los gastos totales & $5,50 \%$ & $7,00 \%$ & $6,30 \%$ & $7,10 \%$ \\
\hline RCR como \% de los gastos totales & $6,00 \%$ & $6,50 \%$ & $6,60 \%$ & $6,70 \%$ \\
JHH como \% de los gastos totales & $5,40 \%$ & $6,60 \%$ & $5,70 \%$ & $6,60 \%$ \\
JHM como \% de los gastos totales & $6,30 \%$ & $7,60 \%$ & $7,30 \%$ & $7,70 \%$ \\
\hline
\end{tabular}

Fuente: Elaboración propia, con datos de la Encuesta Presupuestos Familiares, del año 2012.

hogar. Por otro lado, al comparar el gasto en salud como porcentaje de los gastos totales entre las zonas geográficas, encontramos que tanto por hogar y PC es mayor el índice en el RCR para los asegurados del FONASA, mientras que para los afiliados en ISAPRE es mayor el porcentaje en el GS. Este indicador también es mayor para el JHM en el análisis por hogar y PC en ambos seguros.

\section{Evolución de la protección financiera}

Al analizar la evolución de la protección financiera a NN entre los años 2007 y 2012 (Tabla 3), encontramos que el GB aumentó 14,12\%, mientras que el GB como porcentaje de los gastos totales por hogar creció desde 5,6\% a 6,2\%. Además, estadísticamente, no hubo aumento del GB por hogar en el GS, aumentando si en el RCR (Tabla 3). Cabe mencionar que los resultados del gasto de bolsillo PC presentan la misma tendencia anterior (Tabla 4).

Respecto a la medición de la equidad financiera, el IG del GB hogar y PC presentó una disminución entre la EPF 2007 y la 2012, tanto a NN, GS y del RCR (Tablas 3 y 4 ).

Con relación al GB por quintiles, se observa un aumento estadístico del GB por hogar a NN (Tabla 5) en el quinto quintil y en el GS en el segundo quintil, mientras que para el RCR, aumentó en los quintiles 1, 3 y 5. Para el gasto de bolsillo PC, se observan cambios estadísticamente significativos en el segundo quintil a NN y en el GS y en los quintiles 3 y 5 para el RCR.

Por otro lado, el cociente del GB entre el quintil de mayores y el de menores ingresos, se incrementó entre la EPF 2007 y 2012, a NN, GS y RCR tanto a nivel de hogares como PC. 
Tabla 3. Gasto de bolsillo en salud y coeficiente Gini por hogares, a nivel nacional, del Gran Santiago y del resto de capitales regionales, Chile (\$ de 2015)

\begin{tabular}{|lcccccc|}
\hline Geografía & \multicolumn{2}{c}{ Nacional } & \multicolumn{2}{c|}{ Gran Santiago } & \multicolumn{2}{c|}{ Resto capitales } \\
Gasto/años & $\mathbf{2 0 0 7}$ & $\mathbf{2 0 1 2}$ & $\mathbf{2 0 0 7}$ & $\mathbf{2 0 1 2}$ & $\mathbf{2 0 0 7}$ & $\mathbf{2 0 1 2}$ \\
\hline Gasto bolsillo en salud por hogar & 48.223 & 55.031 & 54.548 & 54.874 & 38.086 & 55.246 \\
\hline p value & \multicolumn{2}{c}{0,0038} & \multicolumn{2}{c}{0,9261} & & 0,0000 \\
\hline Gasto bolsillo por hogar (como \% del gasto total) & $5,6 \%$ & $6,2 \%$ & $5,8 \%$ & $6,3 \%$ & $5,1 \%$ & $6,2 \%$ \\
\hline GINI gasto bolsillo en salud por hogar & 0,81 & 0,77 & 0,82 & 0,78 & 0,80 & 0,75 \\
\hline
\end{tabular}

Fuente: Elaboración propia, con datos de la Encuesta Presupuestos Familiares, de los años 2007 y 2012.

Tabla 4. Gasto de bolsillo en salud y coeficiente Gini por per cápita, a nivel nacional, del Gran Santiago y del resto de capitales regionales, Chile (\$ de 2015)

\begin{tabular}{|lcccccc|}
\hline Geografía & \multicolumn{2}{c}{ Nacional } & \multicolumn{2}{c|}{ Gran Santiago } & \multicolumn{2}{c|}{ Resto capitales } \\
Gasto/años & $\mathbf{2 0 0 7}$ & $\mathbf{2 0 1 2}$ & $\mathbf{2 0 0 7}$ & $\mathbf{2 0 1 2}$ & $\mathbf{2 0 0 7}$ & $\mathbf{2 0 1 2}$ \\
\hline Gasto bolsillo en salud per cápita & 17.248 & 19.683 & 19.969 & 19.869 & 12.888 & 19.428 \\
\hline p value & \multicolumn{2}{c|}{0,0177} & \multicolumn{2}{c}{0,9489} & & 0,0000 \\
\hline Gasto bolsillo per cápita (como \% del gasto total) & $6,0 \%$ & $6,7 \%$ & $6,4 \%$ & $6,8 \%$ & $5,3 \%$ & $6,6 \%$ \\
\hline GINI gasto bolsillo en salud por hogar & 0,83 & 0,79 & 0,84 & 0,81 & 0,81 & 0,77 \\
\hline
\end{tabular}

Fuente: Elaboración propia, con datos de la Encuesta Presupuestos Familiares, de los años 2007 y 2012.

Tabla 5. Gasto de bolsillo en salud (hogar y per cápita) por quintiles de ingreso total, a nivel nacional, del Gran Santiago y del resto de capitales regionales, Chile (\$ de 2015)

\begin{tabular}{|c|c|c|c|c|c|c|c|c|c|c|c|c|}
\hline \multirow{2}{*}{$\begin{array}{l}\text { Tipo de gasto } \\
\text { Geografía } \\
\text { Quintiles/ } \\
\text { años }\end{array}$} & \multicolumn{4}{|c|}{$\begin{array}{c}\text { Gasto hogar } \\
\text { Gran } \\
\text { Santiago }\end{array}$} & \multicolumn{2}{|c|}{$\begin{array}{l}\text { Resto } \\
\text { capitales }\end{array}$} & \multicolumn{4}{|c|}{$\begin{array}{c}\text { Gasto per cápita } \\
\text { Gran } \\
\text { Santiago }\end{array}$} & \multicolumn{2}{|c|}{$\begin{array}{c}\text { Resto } \\
\text { capitales }\end{array}$} \\
\hline & 2007 & 2012 & 2007 & 2012 & 2007 & 2012 & 2007 & 2012 & 2007 & 2012 & 2007 & 2012 \\
\hline Quintil 1 & 12.785 & 15.331 & 14.337 & 15.530 & 10.863 & 14.955 & 3.579 & 4.002 & 4.175 & 4.128 & 2.841 & 3.765 \\
\hline$p$-value & \multicolumn{2}{|c|}{0,1294} & \multicolumn{2}{|c|}{0,6344} & \multicolumn{2}{|c|}{0,0212} & \multicolumn{2}{|c|}{0,4579} & \multicolumn{2}{|c|}{0,9583} & \multicolumn{2}{|c|}{0,0629} \\
\hline Quintil 2 & 22.175 & 26.750 & 19.519 & 26.821 & 25.800 & 26.643 & 6.219 & 7.588 & 5.882 & 7.353 & 6.678 & 7.942 \\
\hline$p$-value & \multicolumn{2}{|c|}{0.0776} & \multicolumn{2}{|c|}{0.0052} & \multicolumn{2}{|c|}{0.8644} & \multicolumn{2}{|c|}{0.0488} & \multicolumn{2}{|c|}{0.0499} & \multicolumn{2}{|c|}{0.3260} \\
\hline Quintil 3 & 36.751 & 37.066 & 41.187 & 33.956 & 29.254 & 41.488 & 11.181 & 11.782 & 12.398 & 10.757 & 9.863 & 13.238 \\
\hline$p$-value & \multicolumn{2}{|c|}{0,9419} & \multicolumn{2}{|c|}{0,2611} & \multicolumn{2}{|c|}{0,0036} & \multicolumn{2}{|c|}{0,6758} & \multicolumn{2}{|c|}{0,4427} & \multicolumn{2}{|c|}{0,0032} \\
\hline Quintil 4 & 57.104 & 62.038 & 55.970 & 61.301 & 58.993 & 62.767 & 18.563 & 21.209 & 17.565 & 20.691 & 20.224 & 21.722 \\
\hline$p$-value & \multicolumn{2}{|c|}{0,2811} & \multicolumn{2}{|c|}{0,4235} & \multicolumn{2}{|c|}{0,5823} & \multicolumn{2}{|c|}{0,0939} & \multicolumn{2}{|c|}{0,1373} & \multicolumn{2}{|c|}{0,5841} \\
\hline Quintil 5 & \multicolumn{2}{|c|}{112.299134 .464} & 126.389 & 148.684 & 80.119 & 116.789 & 46.700 & 54.054 & 53.219 & 61.188 & 31.810 & 45.185 \\
\hline$p$-value & \multicolumn{2}{|c|}{0,0119} & \multicolumn{2}{|c|}{0,0950} & \multicolumn{2}{|c|}{0,0000} & \multicolumn{2}{|c|}{0,0918} & \multicolumn{2}{|c|}{0,2112} & \multicolumn{2}{|c|}{0,0002} \\
\hline Razón (Q5/Q1) & 8,78 & 10,52 & 9,89 & 11,63 & 6,27 & 9,13 & 13,05 & 13,51 & 12,75 & 14,82 & 11,20 & 12,00 \\
\hline
\end{tabular}

Fuente: Elaboración propia, con datos de la Encuesta Presupuestos Familiares, de los años 2007 y 2012. 


\section{Discusión}

La protección financiera de los beneficiarios de FONASA es 3,8 veces superior, en términos absolutos, por hogar y PC respecto a los de ISAPRE, lo que se reflejaría en la mayor proporción del gasto total de los beneficiarios de ISAPRE destinado a GB en prestaciones de salud. Estos se deberían a una mezcla entre la cantidad de prestaciones, sus precios y las bonificaciones de los seguros ${ }^{17}$. Igualmente, la frecuencia de uso de prestaciones de salud (consultas, controles, exámenes y cirugías), según la Encuesta de Caracterización Socioeconómica Nacional (CASEN) 2011 -representativa a nivel nacional, regional, urbano y rural y comunal ${ }^{18}$-, es menor para los beneficiarios del FONASA $^{19}$. Respecto a los precios, no se cuenta con información pública de un índice de precios comparable entre los seguros, por lo que no es posible colegir. En la bonificación, los datos de la Superintendencia de Salud indicaban para el año 2012 que el GB promedio por prestaciones para los beneficiarios de ISAPRE fue de $33,7 \%$, sin considerar seguros complementarios, desconociéndose este indicador para el FONASA.

A nivel geográfico, estadísticamente, en promedio los beneficiarios del FONASA realizan un GB mayor en el RCR que en el GS. Una hipótesis para explicarlo, sería que la oferta de profesionales de la salud es menor en regiones, lo cual disminuiría el poder de negociación del seguro público con dichos prestadores y generaría hipotéticamente mayores precios y menor acceso a prestaciones. Esto se ratificaría con los datos de FONASA en su Modalidad de Libre Elección (permite a los cotizantes del FONASA y su familia acceder a prestadores privados con convenio) $)^{20}$, los que muestran una mayor tasa de beneficiarios por profesionales inscritos en regiones que en la capital. Esto es preocupante para los beneficiarios del FONASA, ya que al ser menores los ingresos promedios por hogar en regiones, de acuerdo a los datos del Ministerio de Desarrollo Social $(\mathrm{MIDESO})^{21}$, facilita que su GB por hogar en salud como porcentaje de los gastos totales supere a los del GS (Tabla 2).

Con respecto al análisis de género del jefe de hogar, no se encontraron diferencias entre $\mathrm{JHH}$ y JHM al analizar el GB por hogar en ISAPRE y FONASA. Sin embargo, el GB PC y GB como porcentaje del gasto total es mayor en aquellos hogares cuyo jefe de hogar es mujer. Esto sería válido para afiliadas de ambos seguros, implicando que la protección financiera es menor para dichos hogares, los cuáles según el MIDESO, para los años 2009 y 2013, son aquellos con una mayor proporción de pobreza y con menores niveles de ingreso por hogar ${ }^{22}$.

La evolución del GB mostró un aumento, a nivel de hogares y PC, a NN y en el RCR. Esto se reflejaría, en un incremento en el GB por hogar a $\mathrm{NN}$ en el quinto quintil, en el RCR en el primer, tercero y quinto quintil de ingreso y en el GS en el segundo quintil. Esto revelaría un problema de protección financiera en los seguros, ya que, ceteris paribus, aumentaría la proporción del costo de las prestaciones financiadas directamente por las personas y, consiguientemente, su riesgo financiero.

Otra evidencia son los resultados de la encuesta CASEN, para años similares a los de la EPF, en 3 prestaciones, mostrando un aumento del porcentaje de beneficiarios del grupo A del FONASA que realizaron $\mathrm{GB}$, grupo en el cual por definición institucional se espera que su población no lo realice. A saber, las consultas médicas generales, las consultas por especialidad y las hospitalizaciones pasaron desde $5,14 \%$ a $8,40 \%, 12,73 \%$ a $21,49 \%$ y $4,27 \%$ a $26,96 \%$, respectivamente, de los beneficiarios que debieron realizar gasto de bolsillo entre los años 2006 y el $2013^{19}$.

Además, desde la perspectiva de equidad, si bien aumentó estadísticamente el GB en prestaciones de salud en los hogares de mayores recursos, lo que se habría observado en un incremento de la relación entre el quinto y el primer quintil, la distribución del GB fue de manera más proporcional entre los hogares, observado por una disminución del IG, obteniéndose un resultado contradictorio y no concluyente respecto a la evolución de la equidad.

Una limitación del estudio fue la imposibilidad de obtener información sobre el GB de los hogares como porcentaje de los gastos totales en salud, debido a que las EPF analizadas registran los pagos realizados efectivamente por los hogares en prestaciones. Solo en el caso de los afiliados a ISAPRE es factible estimar su GB, que, en promedio, pasa desde $32,9 \%$ a 33,7 entre el año 2007 y el $2012^{23}$. Esto es especialmente relevante para los beneficiarios de FONASA que acceden a prestaciones de salud en la red pública, donde la protección financiera entregada por el FONASA a través de la 
Modalidad de Atención Institucional es mayor ${ }^{24}$. Por lo tanto, para medir con mayor precisión la cobertura financiera de los seguros sería deseable que la EPF recabe el gasto total en prestaciones de salud, tanto de aquellas que conllevan GB como las que no significan efectuarlo. Esto probablemente llevaría a tener una mejor estimación de la protección financiera de los seguros.

Finalmente, se pudo establecer que hubo un aumento del GB entre los años en estudio y que existen problemas de desigualdad en cobertura por parte de las aseguradoras. Es deseable que futuras investigaciones analicen la evolución de los efectos precio, cantidad y bonificación del gasto en servicios de salud (incluidos los fármacos) para determinar su impacto individual y combinado sobre el GB de las familias.

\section{Referencias}

1. Larrañaga O. Eficiencia y equidad en el sistema de salud chileno. Serie del Financiamiento del Desarrollo 49, CEPAL 1997.

2. Goic A. El sistema de salud de Chile: una tarea pendiente. Rev Med Chile 2015; 143: 774-86.

3. Cid C, Aguilera X, Arteaga O, Barría S, Barría P, Castillo C, et al. Informe Final, Comisión Asesora Presidencial para el Estudio y Propuesta de un Nuevo Régimen Jurídico para el Sistema de Salud Privado. Comisión Presidencial ISAPRES. Decreto No 71 del 14 de abril de 2014. p. 27-28. Disponible en www.minsal. cl [consultado el 29 de noviembre de 2016].

4. Drago M. La reforma al sistema de salud chileno desde la perspectiva de los derechos humanos. Serie de Políticas Sociales CEPAL, Naciones Unidas 2006; 121 : 28-30

5. Uthoff, Sánchez \& Campusano. El mercado de seguros de salud: Lecciones sobre el conflicto entre equivalencia y solidaridad. Revista de la CEPAL 2012; 108: 11.

6. Fondo Nacional de Salud. Estadística Institucional, Estadística Demográfica. FONASA 2014. Disponible en www.fonasa.cl [consultado el 15 de mayo de 2017].

7. Organización Mundial de la Salud. Informe sobre la salud en el mundo 2000, Mejorar el desempeño del sistema de salud. Ginebra, Suiza: OMS 2000. Disponible en www.who.int [consultado el 1 de junio de 2017].

8. Organización Mundial de la Salud. Informe sobre la salud en el mundo 2010, Financiación de los sistemas de salud: el camino hacia la cobertura universal. Ginebra,
Suiza: OMS 2010. Disponible en www.who.int [consultado el 1 de junio de 2017].

9. Wang H, Switlick K, Ortiz C, Zurita B, Connor C. Health Insurance Handbook, How to Make It Work. World Bank Working 2012; Paper No. 219.

10. Cutler D, Zeckhauser R. The Anatomy of Health Insurance. Handbook of Health Economics 2000; Volume 1A, Charter 11. Harvard University and National Bureau of Economic Research.

11. Moreno R, Thomson S, Xu K. Measuring and comparing financial protection. European Observatory on Health Systems and Policies Series 2013. Chapter 8. Health system performance comparison, an agenda for policy, information and research.

12. Instituto Nacional de Estadísticas. Encuesta de Presupuestos Familiares (EPF) 2012. Base de datos INE 2013. Disponible en www.ine.cl [consultado el 29 de noviembre de 2016].

13. Instituto de Administración de Salud. Informe Final Evaluación del Gasto Institucional del Fondo Nacional de Salud (FONASA). Preparado para la Dirección de Presupuestos, Ministerio de Hacienda 2013. Disponible en www.dipres.gob.cl [consultado el 26 de noviembre de 2016].

14. Xu K, et al. Exploring the thresholds of health expenditure for protection against financial risk. World Health Report 2010, Background Paper, 19 Geneva.

15. Instituto Nacional de Estadísticas. VII Encuesta de Presupuestos Familiares. Resultados VII EPF. INE 2013. Disponible en www.ine.cl [consultado el 29 de noviembre de 2016].

16. StataCorp. Stata Base Reference Manual: Release 13. Statistical Software. College Station, TX: StataCorp LP 2013. Disponible en www.stata.com [consultado el 19 de junio de 2017].

17. Instituto Nacional de Estadísticas (sin año). Metodología para la elaboración de indicadores referenciales de costos de las Instituciones de Salud Previsional-ISAPRE. Disponible en www.ine.cl [consultado el 4 de septiembre de 2017].

18. Fondo Nacional de Salud. Estadística Institucional, Prestadores de Atenciones de Salud. FONASA 2013. Disponible en www.fonasa.cl [consultado el 29 de noviembre de 2016].

19. Ministerio de Desarrollo Social. Evolución y distribución del ingreso de los hogares (2006-2013), Síntesis de Resultados. MIDESO 2013. Disponible en www. ministeriodesarrollosocial.gob.cl [consultado el 29 de noviembre de 2016].

20. Ministerio de Desarrollo Social. Encuesta de Caracterización Socioeconómica Nacional (CASEN). Género, 
síntesis de resultados. MIDESO 2013. Disponible en www.ministeriodesarrollosocial.gob.cl [consultado el 1 de enero de 2018].

21. Ministerio de Desarrollo Social. Encuesta de Caracterización Socioeconómica Nacional (CASEN). Archivo histórico de encuesta CASEN. MIDESO 2013. Disponible en www.ministeriodesarrollosocial.gob.cl [consultado el 29 de noviembre de 2016].

22. Ministerio de Desarrollo Social. Encuesta de Caracterización Socioeconómica Nacional (CASEN). Bases de datos 2006-2013. MIDESO 2013. Disponible en www. ministeriodesarrollosocial.gob.cl [consultado el 29 de noviembre de 2016].

23. Superintendencia de Salud. Boletines Estadísticos. Biblioteca Digital. Disponible en www.supersalud.gob.cl [consultado el 1 de mayo de 2017].

24. Fondo Nacional de Salud. Beneficiarios-FONASA, Información General del Seguro, Tramos y Copagos. FONASA 2018. Disponible en www.fonasa.cl [consultado el 1 de enero de 2018]. 